\title{
Evaluation of the association between the first observation and the longitudinal change in C-reactive protein, and all-cause mortality
}

\author{
C J Currie, ${ }^{1}$ C D Poole, ${ }^{2}$ P Conway ${ }^{3}$
}

${ }^{1}$ Department of Medicine, School of Medicine, Cardiff University, Cardiff, UK;

${ }^{2}$ Pharmatelligence, Cardiff, UK;

${ }^{3}$ Health Economics, Wyeth

Europa Limited, Maidenhead, UK

Correspondence to:

Dr C Currie, The Pharma

Research Centre, University

Hospital of Wales, Medicentre,

Cardiff CF14 4UJ, UK; currie@

cardiff.ac.uk

Accepted 31 July 2007

Published Online First

29 August 2007

\begin{abstract}
Objective: To evaluate the association between vascular inflammation as measured by subacute $\mathrm{C}$-reactive protein (CRP; 1-10 mg/l) and all-cause mortality and the association between change in CRP status (normal $\leqslant 3 \mathrm{mg} / \mathrm{l}$ and elevated $>3 \mathrm{mg} / \mathrm{l}$ ) and all-cause mortality. Methods: Probabilistic record linkage was used to match hospital episode data, laboratory reports and mortality statistics in a large urban population. Survival was evaluated using Cox proportional hazards regression models.
\end{abstract}

Results: 22962 patients had their first CRP measurement in the subacute range (1-10 mg/l). Analysis grouped by each additional unit increase in CRP across the subacute range was associated with a $7.3 \%(95 \% \mathrm{Cl}$ $5.4 \%$ to $9.2 \%$ ) increase in the hazard ratio (HR) of death over 4 years, after controlling for confounding factors $(p<0.001)$. Repeated CRP observations around 1 year apart were recorded in 5811 subjects. After controlling for confounding factors, in patients whose CRP changed from normal $(\leqslant 3 \mathrm{mg} / \mathrm{l})$ to elevated ( $>3 \mathrm{mg} / \mathrm{l})$, the HR increased 6.7-fold $(p<0.001)$ relative to cases whose CRP remained normal. By comparison, among those subjects whose CRP was reduced from elevated to normal, the hazard ratio halved to $3.5(p=0.018)$. In an underpowered analysis of time to cardiovascular events, an identical pattern of risk emerged.

Conclusions: CRP level predicted all-cause mortality, and additional inclusion of prior change in CRP level and current CRP level more so. Increasing vascular inflammation, as measured by CRP, increases the likelihood of death.

C-reactive protein (CRP) is a highly conserved, five-subunit protein produced largely in the liver. It is thought to be an acute-phase reactant to tissue damage, infection, inflammation and malignancy. ${ }^{1}$ The role of CRP in the development of cardiovascular disease has come under considerable scrutiny following epidemiological evidence of an association between elevated CRP and the prevalence of atherosclerotic vascular disease and risk of both first and subsequent cardiovascular events. ${ }^{2}$ There is little doubt that CRP adds to traditional risk factors in the global assessment of cardiovascular risk, and it may be at least as important as hypertension in prediction of outcome. ${ }^{3}$ In the USA, doctors are now recommended to measure high-sensitivity CRP in asymptomatic people with an intermediate risk of coronary heart disease to optimise their assessment of cardiovascular risk. ${ }^{4}$

The hypothesis that CRP is an important marker of atherothrombosis has now become the focus of intense investigation. ${ }^{5}$ This theory has considerable clinical significance as CRP levels are modifiable. Although there is not yet unanimous agreement over the pro-atherogenic role of $\mathrm{CRP}^{2}$, supporting evidence is emerging. ${ }^{6}$ There is considerable experimental data linking CRP to endothelial dysfunction, vascular remodelling and key stages of the atherothrombotic process. Exogenous CRP also activates both inflammation and coagulation when administered to healthy volunteers, ${ }^{7}$ an effect independent of endotoxin or other contaminants. ${ }^{6}$

Resolution of this hypothesis is of particular interest in the management of autoimmune conditions where chronic elevation of CRP accelerates atherosclerosis ${ }^{8}$ and, thereby potentially increases mortality. Although CRP has been shown to be a poor predictor of the incidence of rheumatoid arthritis, ${ }^{9}$ it is central to the evaluation of disease progression and response to therapeutic intervention $^{10-12}$ and increasingly suspected as a proatherogenic agent in affected patients. ${ }^{13-15}$ Similar associations are evident in psoriasis, ${ }^{16}$ where the relative risk of myocardial infarction in young patients with severe disease is elevated threefold over disease-free controls after adjustment for relevant baseline cardiovascular risk factors. ${ }^{17}$

Despite the large body of epidemiological data relating to $\mathrm{CRP}$, few studies have examined the relationship between longitudinal change in CRP and prognosis. ${ }^{18}$ In this study, we sought to examine the effect of CRP change on both allcause mortality and cardiovascular morbidity using routine laboratory data collected in hospital practice, following validation of the association between snapshot CRP measurement and all-cause mortality within the available data.

\section{PATIENTS AND METHODS}

These data represented hospital inpatient admissions, laboratory investigations, and mortality data for the population resident in the geographical area of Cardiff and the Vale of Glamorgan (UK), a predominantly urban area with a population of about 424000 people. ${ }^{19}$ The epidemiology of heart disease and other coronary-related epidemiology and survival have been described in this population using the same methods as those described here. ${ }^{20} 21$ These data were linked by proprietary software using validated probabilistic matching algorithms on personal identifiers. ${ }^{22}$ Following recommendation from the Patient Information Advisory Group to the Secretary of State for Health an exemption from the requirement for explicit consent was granted under section 60 of the Health and Social 
Table 1 Cox proportional hazards regression model for all-cause mortality as a function of subacute first C-reactive protein (CRP) observation

\begin{tabular}{|c|c|c|c|c|c|c|}
\hline \multirow[b]{2}{*}{ Variables in the equation } & \multirow[b]{2}{*}{ B } & \multirow[b]{2}{*}{ SE } & \multirow[b]{2}{*}{$\mathbf{p}$} & \multirow[b]{2}{*}{ HR } & \multicolumn{2}{|c|}{$95 \% \mathrm{Cl}$ for $\exp (\mathrm{B})$} \\
\hline & & & & & Lower & Upper \\
\hline \multicolumn{7}{|l|}{ Demographics } \\
\hline Age at 1 st subacute CRP & 0.062 & 0.002 & $<0.001$ & 1.064 & 1.060 & 1.068 \\
\hline Male gender & 0.205 & 0.049 & $<0.001$ & 1.228 & 1.115 & 1.351 \\
\hline \multicolumn{7}{|l|}{ CRP measurement } \\
\hline 1st CRP & 0.071 & 0.009 & $<0.001$ & 1.073 & 1.054 & 1.092 \\
\hline \multicolumn{7}{|l|}{ Prior diagnoses } \\
\hline Prior cancer & 0.403 & 0.070 & $<0.001$ & 1.497 & 1.305 & 1.717 \\
\hline Prior diabetes & 0.398 & 0.067 & $<0.001$ & 1.488 & 1.305 & 1.697 \\
\hline Prior vascular disease & 0.331 & 0.051 & $<0.001$ & 1.393 & 1.261 & 1.538 \\
\hline
\end{tabular}

Care Act 2001. Ethical approval was granted by the Bro Taf local research ethics committee.

\section{Hospital episode data}

Hospital episode data were obtained from the national NHS hospital episode collating body for Wales. Inpatient data were available for 1500876 finished consultant episodes representing 1326211 admissions for 477841 subjects spanning a period of 13 years to February 2005. Clinical diagnoses were recorded using both ICD-9, which predominated until 1995, and was then phased out and replaced with ICD-10.

\section{Mortality data}

Mortality data were obtained from the Office of National Statistics for subjects resident in South Glamorgan at the time of death. Over a period of 12 years to March 2005, there were 50963 deaths among local residents. Cause of death was classified by ICD-9 until 2000, when phased out by ICD-10.

\section{Laboratory data}

All laboratory results originated from the Cardiff and Vale NHS Trust Laboratory Service covering a 5 -year period to January 2006. Over this time some 43265603 tests were reported for 295631 subjects

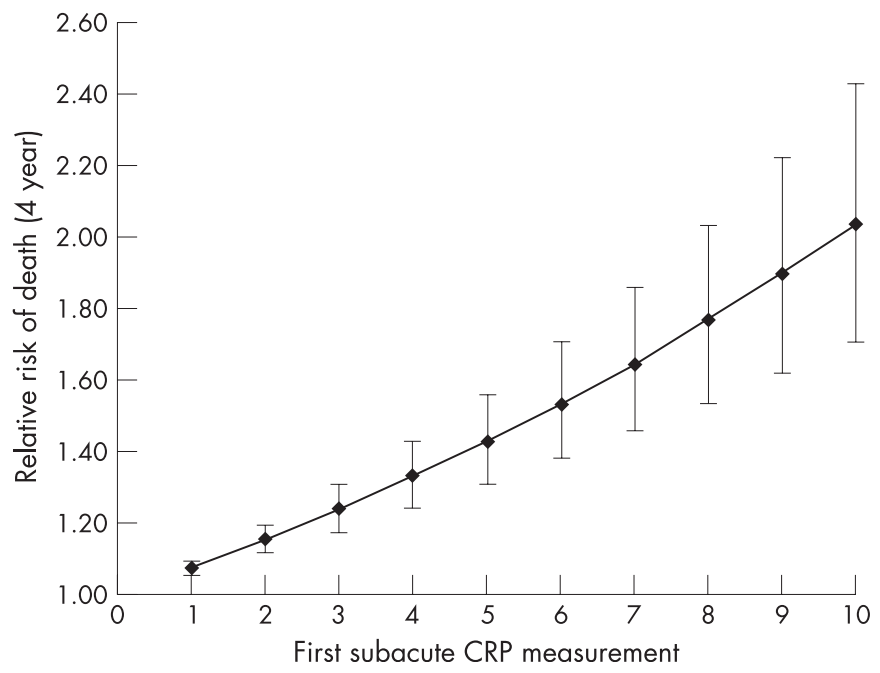

Figure 1 Interpolated relative risk of death as a function of subacute first C-reactive protein (CRP) measurement.
The key biochemical marker in this investigation, serum CRP concentration, was measured at the University of Wales Hospital Department of Laboratory Medicine using a Behring latex-enhanced nephelometer analyser system. As the assay was not of high sensitivity it was decided to categorise the CRP level of subjects according to the scientific statement recently issued by the American Heart Association and Centers for Disease Control and Prevention, ${ }^{23}$ a method used previously in large epidemiological studies. ${ }^{24}$ An elevated level of CRP was defined as $>3 \mathrm{mg} / \mathrm{l}$ while levels $>10 \mathrm{mg} / \mathrm{l}$ were defined as representing acute inflammatory processes. In total there were 432272 CRP observations from 98505 people.

Measurement of serum lipids over the same period was performed by the same department and yielded 315178 total cholesterol (TC) observations from 87512 people in addition to 124721 triglyceride results; 93215 low-density lipoprotein (LDL) observations; 50284 high-density lipoprotein (HDL) observations; and 79538 reported TC/HDL ratio observations.

\section{Case selection}

Patients regarded as having a subacute 1st CRP observation were selected if their first CRP measurement was in the range 1$10 \mathrm{mg} / \mathrm{l}$. Cases where CRP change was recorded were included if at least two observations had been recorded about 1 year apart. Given high intraindividual variability for CRP, ${ }^{25}{ }^{26}$ the baseline (2nd observation) CRP level was calculated as the average of all observations within 90 days of the first CRP observation. Similarly, the baseline CRP level was defined as the mean of values occurring within a further 90 days of the second (1 year) observation. CRP change was then defined for the period as normal $\rightarrow$ normal $\quad(\mathrm{NN}), \quad$ normal $\rightarrow$ elevated $(\mathrm{NE})$, elevated $\rightarrow$ normal $(\mathrm{EN})$, and elevated $\rightarrow$ elevated $(\mathrm{EE})$.

\section{Disease ascertainment}

Acute ischaemic events were defined as any admission or cause of death which included the diagnostic codes for angina pectoris (ICD-10, I20*; ICD-9, 413), acute myocardial infarction (I21*, 410), subsequent myocardial infarction (I22*), other acute ischaemic heart disease (I24*, 411), cerebral infarction (I63*, 434) and stroke (I64*, V171). A prior diagnosis of cancer was defined as any admission before the first CRP measurement that included a diagnostic code for malignant neoplasm (ICD-10, C00*-C97*). Although ICD-9 codes were not examined, those patients whose last such admission was before 1996 were assumed to have been cured of their cancer. Prior vascular diagnosis was defined as any admission before the first CRP 
Table 2 Patient characteristics by their first C-reactive protein (CRP) observation

\begin{tabular}{|c|c|c|c|c|c|}
\hline Characteristics & All subjects & $\begin{array}{l}\text { Normal CRP } \\
\leqslant 3 \mathrm{mg} / \mathrm{l}\end{array}$ & $\begin{array}{l}\text { Elevated CRP } \\
4-10 \mathrm{mg} / \mathrm{l}\end{array}$ & $\begin{array}{l}\text { Acutely high CRP } \\
>10 \mathrm{mg} / \mathrm{l}\end{array}$ & p Value \\
\hline Number of subjects (n) & 5811 & 828 & 1531 & 3452 & \\
\hline Age (years), mean (SD) & $61.3(20.29)$ & $56.2(21.8)$ & $59.9(19.8)$ & $63.1(19.9)$ & ANOVA $<0.001$ \\
\hline \multicolumn{6}{|l|}{$\chi^{2}<0.001$} \\
\hline Total cholesterol (mmol/l), mean (SD) & $4.7(1.4)$ & $4.7(1.2)$ & $4.8(1.5)$ & $4.7(1.3)$ & ANOVA 0.186 \\
\hline LDL (mmol/l), mean (SD) & $2.9(1.0)$ & $3.1(0.8)$ & $2.9(1.0)$ & $2.8(1.1)$ & ANOVA 0.291 \\
\hline With prior cancer (\%) & 8.7 & 6.3 & 7.2 & 9.9 & LLA 0.001 \\
\hline Dead within 3 years of CRP observation period (\%) & 13.2 & 9.1 & 12.1 & 20.0 & LLA $<0.001$ \\
\hline $\begin{array}{l}\text { Experiencing acute ischaemic event within } 3 \text { years } \\
\text { of CRP observation (\%) }\end{array}$ & 2.7 & 1.7 & 3.3 & 2.7 & LLA 0.339 \\
\hline
\end{tabular}

ANOVA, one-way analysis of variance; $\chi^{2}$, chi-square test; LDL, low-density lipoprotein; LLA, linear-by-linear association.

measurement that included a diagnostic code for any disease of the circulatory system (ICD-10, I00*-I99*). Prior diabetes diagnosis was defined as any admission before the first CRP measurement that included a diagnostic code for diabetes mellitus (ICD-10, E10*-E14*).

\section{Statistical methods}

Survival was evaluated using Cox proportional hazards regression model (CPHM), using SPSS version 14 (SPSS Inc, Chicago, USA). Independent covariates of both survival and time to first acute ischaemic event were determined within the models following the appropriate CRP observation. Patients who were admitted with a diagnosis of an acute ischaemic event during the CRP observation period were excluded from the analysis. Time to event was measured from the date of the last reported CRP observation. Post hoc power analysis (StudySize version 2.0.1, CreoStat) for mortality indicated that CRP-change category membership would detect a minimum hazard ratio (HR) in the range 1.71-1.34 (normal-normal and elevatedelevated, respectively) with a 95\% probability and $90 \%$ power. Under the same statistical conditions, CRP-change categories were able to detect HRs for acute ischaemic events in the range 2.97-1.83 (normal-normal and elevated-elevated, respectively).

\section{RESULTS}

\section{Subacute first CRP measurement}

Among all patients with at least one CRP measurement, 22962 had a subacute value at their first observation. Their mean age was 55 years and $46 \%$ were male. Within this cohort there were 479 deaths $(2.1 \%)$ of any cause within a follow-up period of
4 years. CPHM showed each unit increase in CRP within the subacute range resulted in a $7.3 \%$ increase in the $\mathrm{HR}(95 \% \mathrm{CI}$ $5.4 \%$ to $9.2 \%$ ) after controlling for age, gender, prior cancer, prior diabetes and pre-existing vascular disease (table 1). Graphical interpolation of this relationship (fig 1) shows the mean increased relative risk of death in patients with a CRP of $10 \mathrm{mg} / \mathrm{l}$ to be nearly double that of patients with the lowest CRP measurement (1 mg/l).

\section{CRP change}

Repeated CRP observations at 1 year were available for 5811 subjects. Among these, the majority (59\%) had a first quarter average CRP in the acute range $(>10 \mathrm{mg} / \mathrm{l}), 26 \%$ had an elevated but subacute CRP level (4-10 mg/l), while the remainder (14\%) had a normal CRP level ( $\leqslant 3 \mathrm{mg} / \mathrm{l})$. Subjects with acute CRP were significantly older than patients with either elevated or normal CRP (63.1 years vs 59.9 years and 56.2 years, respectively; $p<0.001$ ) and had increased prevalence of diabetes $(11.6 \%$ vs $8.8 \%$ and $8.9 \%$, respectively; $p=0.010$; table 2). Prevalence of prior vascular disease showed a highly significant linear increase across the categories (normal, 29.1\%; elevated 34.4\%; and acute, 40.6\%; $\mathrm{p}<0.001$ ) as did the prevalence of prior cancer (normal, 6.3\%; elevated $7.2 \%$; and acute, $9.9 \%$; $<0.001$ ). Neither mean total cholesterol nor mean LDL cholesterol showed any significant difference between CRP categories ( $p=0.186$ and $p=0.291$, respectively). The likelihood of death within 3 years of the CRP observation period also showed a significant increase across the CRP categories $(\mathrm{p}<0.001)$, almost doubling between normal and acute cases (normal, 9.1\%; elevated, 12.1\%; acute, 20.0\%). A similar linear

Table 3 Patient characteristics by C- reactive protein (CRP) change over 1 year in patients with no acute ischaemic events

\begin{tabular}{|c|c|c|c|c|c|}
\hline Characteristics & Normal $\rightarrow$ normal & Normal $\rightarrow$ elevated & Elevated $\rightarrow$ normal & Elevated $\rightarrow$ elevated & p Value \\
\hline Number of subjects $(\mathrm{n})$ & 335 & 451 & 636 & 3879 & \\
\hline Age (years), mean (SD) & $52.5(20.7)$ & $57.2(22.5)$ & $54.8(21.3)$ & $61.6(19.9)$ & ANOVA $<0.001$ \\
\hline Female (\%) & 55.7 & 53.3 & 59.3 & 56.7 & $\chi^{2}<0.397$ \\
\hline Total cholesterol $(\mathrm{mmol} / \mathrm{l})$, mean (SD) & $4.9(1.1)$ & $4.7(1.1)$ & $4.9(1.1)$ & $4.7(1.5)$ & ANOVA 0.636 \\
\hline LDL (mmol/l), mean (SD) & $3.3(0.8)$ & $2.9(0.7)$ & $2.9(1.0)$ & $3.0(1.0)$ & ANOVA 0.520 \\
\hline With prior diabetes $(\%)$ & 9.5 & 6.9 & 7.4 & 10.0 & $\chi^{2} 0.097$ \\
\hline With prior vascular disease (\%) & 24.1 & 29.3 & 24.0 & 36.5 & $\chi^{2}<0.001$ \\
\hline With prior cancer $(\%)$ & 6.2 & 6.0 & 3.6 & 9.9 & $\chi^{2}<0.001$ \\
\hline $\begin{array}{l}\text { Dead within } 3 \text { years of CRP observation } \\
\text { period }(\%)\end{array}$ & 1.7 & 14.3 & 5.9 & 17.7 & $\chi^{2}<0.001$ \\
\hline $\begin{array}{l}\text { Experiencing acute ischaemic event } \\
\text { within } 3 \text { years of CRP observation (\%) }\end{array}$ & 0.6 & 2.7 & 1.4 & 3.5 & $\chi^{2} 0.001$ \\
\hline
\end{tabular}


Table 4 Cox proportional hazards regression model (CPHM) for all-cause mortality

\begin{tabular}{|c|c|c|c|c|c|c|}
\hline \multirow[b]{2}{*}{ Variables in the equation } & \multirow[b]{2}{*}{$\beta$} & \multirow[b]{2}{*}{ SE } & \multirow[b]{2}{*}{$\mathbf{p}$} & \multirow[b]{2}{*}{ HR } & \multicolumn{2}{|c|}{ 95\% Cl for HR } \\
\hline & & & & & Lower & Upper \\
\hline \multicolumn{7}{|l|}{ Demographics } \\
\hline Male gender & 0.317 & 0.080 & 0.000 & 1.37 & 1.17 & 1.60 \\
\hline Age (years) & 0.058 & 0.003 & 0.000 & 1.06 & 1.05 & 1.07 \\
\hline \multicolumn{7}{|l|}{ Prior diagnoses } \\
\hline Prior vascular disease & 0.208 & 0.083 & 0.012 & 1.23 & 1.05 & 1.45 \\
\hline Prior diabetes & 0.409 & 0.109 & 0.000 & 1.51 & 1.22 & 1.86 \\
\hline Prior cancer & 0.428 & 0.108 & 0.000 & 1.53 & 1.24 & 1.90 \\
\hline \multicolumn{7}{|c|}{ CRP change category (cf normal $\rightarrow$ normal) } \\
\hline Elevated $\rightarrow$ normal & 1.267 & 0.535 & 0.018 & 3.55 & 1.24 & 10.12 \\
\hline Normal $\rightarrow$ elevated & 1.902 & 0.521 & 0.000 & 6.70 & 2.42 & 18.59 \\
\hline Elevated $\rightarrow$ elevated & 2.046 & 0.502 & 0.000 & 7.73 & 2.89 & 20.69 \\
\hline
\end{tabular}

association, however, was not seen in the incidence of acute ischaemic events after the CRP observation period $(p=0.339)$, although the likelihood among elevated cases was approximately twice that of normal cases (3.3\% vs $1.7 \%$; $=0.001)$.

Among this initial cohort, 510 cases experienced an acute ischaemic event during the CRP observation period and were excluded. Of the remainder (5301), the majority of these cases (73.2\%) had consistently elevated CRP (>3 mg/l (EE)); $12.0 \%$ showed a reduction from elevated to normal (EN); $8.5 \%$ had an increased CRP status (NE); and the remaining 6.3\% had stable normal CRP levels (NN; table 3 ). The mean age of EE cases was greater than that of all other categories (61.6 years vs 57.2 years $(\mathrm{NE}), 54.8$ years (EN) and 52.5 years (NN; $\mathrm{p}<0.001)$. Gender balance was similar between CRP-change categories $(p<0.397)$. Prior vascular disease was more prevalent in EE cases $(p<0.001)$, as was prior cancer $(p<0.001)$; however, there was no difference in the prevalence of diabetes at the conventional level of significance $(p=0.097)$. The likelihood of death over 3 years was greater in those with elevated endpoint CRP status $(17.7 \%$ in $\mathrm{EE}$ and $14.3 \%$ in $\mathrm{NE}$ vs $5.9 \%$ in $\mathrm{EN}$ and $1.7 \%$ in $\mathrm{NN}$; $\mathrm{p}<0.001)$. Similarly, for the likelihood of an acute ischaemic event $3.5 \%$ in $\mathrm{EE}$ and $2.7 \%$ in $\mathrm{NE}$ vs $1.4 \%$ in $\mathrm{EN}$ and $0.6 \%$ in $\mathrm{NN} ; \mathrm{p}=0.001)$. Neither mean total cholesterol nor mean LDL cholesterol showed any notable difference between CRP-change categories ( $p=0.636$ and $p=0.520$, respectively).

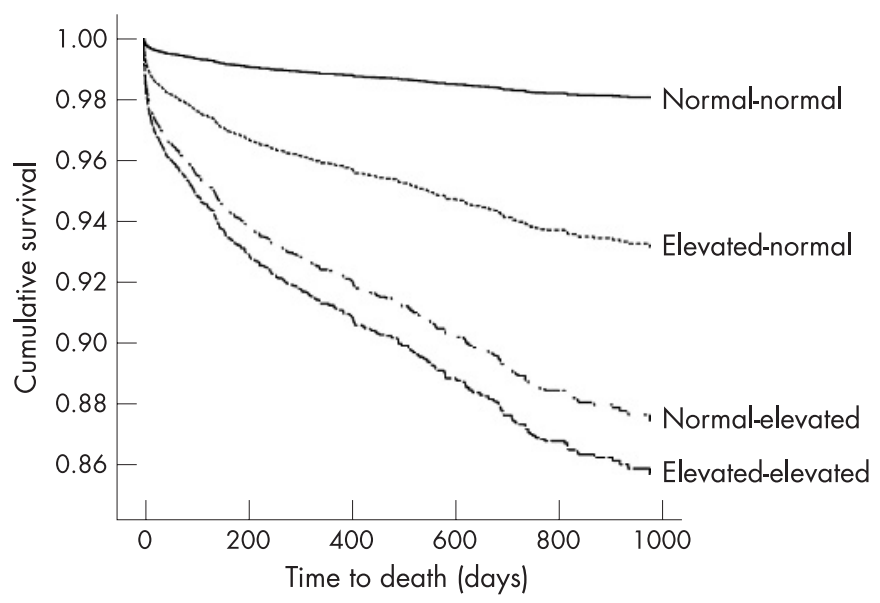

Figure 2 Survival plot for all-cause mortality by CRP-change category standardised for age, sex, prior cancer, prior diabetes, and prior vascular disease.
In a CPHM model of all-cause mortality controlling for age, gender and significant prior diagnoses, the likelihood of death was markedly different between CRP change category (table 4, fig 2). Compared to stable NN cases, the risk of death among increasers (NE) and elevated (EE) cases increased approximately sevenfold $(p<0.001)$, and was twice that of the "decreasers" (EN). Using a similar approach to model time to acute ischaemic events, although not statistically significant, a similar pattern of association emerged (table 5, fig 3).

\section{DISCUSSION}

This study confirmed that increasing levels of subacute CRP were associated with increased risk of 4-year mortality. Other investigators have failed to find an association in the subacute range. ${ }^{27}$ Further, the likelihood of death over 3 years was markedly greater in those whose serum CRP had increased from normal to elevated levels than those whose CRP decreased over a 1-year observation period. The magnitude of the HRs compared with other binary covariates suggested that CRP change was an important marker of mortality in addition to recent CRP status. An identical trend emerged in the longitudinal analysis of acute ischaemic events, limited in this study, most likely, by a lack of statistical power for this specific outcome.

The usefulness of these data was limited by the lack of other vascular risk factors such as blood pressure measurements and obesity. Neither was routinely available from current data collated in secondary care. The limited availability of lipid profiles concomitant to the endpoint CRP observations (TC for 1118 cases and LDL cholesterol for only 241 cases) prevented their meaningful inclusion in these survival analyses. The highly sensitive CRP assay currently now in widespread use enables more accurate detection of lower CRP levels but was not available here for sufficient numbers of patients to allow meaningful analysis of this measure; this did not affect the capacity of these data to investigate the principal research question. ${ }^{28}$ The analysis of cardiovascular event prognosis was undoubtedly limited by statistical power; post hoc power analysis of the available cohort showed a detectable HR outside the relative risks for CRP point estimates. ${ }^{29}{ }^{30}$ Finally, it must be emphasised that this was not a random sample of subjects, but a cohort of patients who had been selected deliberately because a doctor, or another health professional, had requested a CRP measurement. There is a possibility that this selection bias may invalidate the conclusions within this study. However, it was 
Table 5 Cox proportional hazards regression model for an acute ischaemic event following a C-reactive protein (CRP) observation

\begin{tabular}{|c|c|c|c|c|c|c|}
\hline \multirow[b]{2}{*}{ Variables in the equation } & \multirow[b]{2}{*}{$\beta$} & \multirow[b]{2}{*}{ SE } & \multirow[b]{2}{*}{$\mathbf{p}$} & \multirow[b]{2}{*}{ HR } & \multicolumn{2}{|c|}{ 95\% Cl for HR } \\
\hline & & & & & Lower & Upper \\
\hline \multicolumn{7}{|l|}{ Demographics } \\
\hline Male gender & 0.101 & 0.162 & 0.533 & 1.11 & 0.80 & 1.52 \\
\hline Age (years) & 0.042 & 0.006 & $<0.001$ & 1.04 & 1.03 & 1.05 \\
\hline \multicolumn{7}{|l|}{ Prior diagnoses } \\
\hline Prior vascular disease & 0.015 & 0.165 & 0.928 & 1.01 & 0.73 & 1.40 \\
\hline \multicolumn{7}{|c|}{ CRP change category (cf. normal $\rightarrow$ normal) } \\
\hline Elevated $\rightarrow$ normal & 0.767 & 0.782 & 0.327 & 2.15 & 0.46 & 9.97 \\
\hline Normal $\rightarrow$ elevated & 1.046 & 0.769 & 0.174 & 2.85 & 0.63 & 12.85 \\
\hline Elevated $\rightarrow$ elevated & 1.211 & 0.713 & 0.089 & 3.36 & 0.83 & 13.58 \\
\hline
\end{tabular}

felt that because of the large number of observations and subjects, and because of the strength of the association elicited with respect to mortality, that these observations are likely to be valid.

Despite the availability of CRP point estimates for nearly 100000 people, annual follow-up measurements were available for only $5 \%$ of these cases. There may be a case for routine measurement of CRP at intervals, particularly in those with an increased cardiovascular risk status as determined by traditional algorithms and clinical observation. A study of interval measurement of CRP in general practice would form a useful line of further research.

Despite their limitations, these findings do illustrate the striking association between CRP change observed over 1 year and subsequent 4-year all-cause mortality. Among those in whom CRP reduces from an elevated to normal status, the risk of death is halved compared with those whose CRP becomes (or remains) elevated over the observation period. In the context of the weight of evidence supporting the role of CRP as promoter of disease not simply a marker, these findings will be of interest to clinicians in assessing disease prognosis and therapeutic strategies which reduce CRP.

Competing interests: The record-linkage project was originally funded by Diabetes UK. This analysis presented herein was funded by Wyeth Europa Ltd.

Ethics approval: Ethical approval was granted by the Bro Taf local research ethics committee.

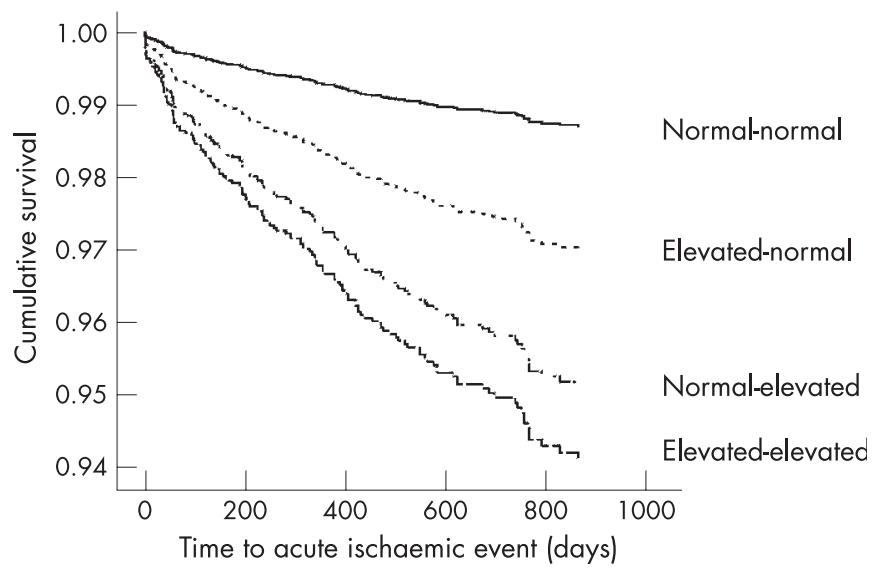

Figure 3 Survival plot for acute ischaemic events by CRP-change category standardised for age, sex, and prior vascular disease.

\section{REFERENCES}

1. Pepys MB, Hirschfield GM. C-reactive protein: a critical update. J Clin Invest 2003:111:1805-12.

2. Scirica BM, Morrow DA. Is C-reactive protein an innocent bystander or proatherogenic culprit? The verdict is still out. Circulation 2006;113:2128-34.

3. Ridker PM, Rifai N, Rose L, et al. Comparison of C-reactive protein and low-density lipoprotein cholesterol levels in the prediction of first cardiovascular events. N Engl J Med 2002;347:1557-65.

4. Pearson TA, Mensah GA, Alexander WR. Markers of inflammation and cardiovascular disease: application to clinical and public health practice a statement for healthcare professionals from the Centers for Disease Control and Prevention and the American Heart Association. Circulation 2003;107:499-511.

5. Nilsson J. CRP: marker or maker of cardiovascular disease? Arterioscler Thromb Vasc Biol 2005;25:1527-8.

6. Verma S, Devaraj $\mathrm{S}$, Jialal I. C-reactive protein promotes atherothrombosis. Circulation 2006;113:2135-50.

7. Bisoendial RJ, Kastelein JJ, Levels $\mathrm{JH}$, et al. Activation of inflammation and coagulation after infusion of C-reactive protein in humans. Circ Res 2005;96:714-6.

8. Abou-Raya A, Abou-Raya S. Inflammation: a pivotal link between autoimmune diseases and atherosclerosis. Autoimmun Rev 2006;5:331-7.

9. Shadick NA, Cook NR, Karlson EW, et al. C-reactive protein in the prediction of rheumatoid arthritis in women. Arch Intern Med 2006;166:2490-4.

10. Kameda H, Sekiguchi N, Nagasawa H, et al. Development and validation of handy rheumatoid activity score with 38 joints (HRAS38) in rheumatoid arthritis patients receiving infliximab. Mod Rheumatol 2006;16:381-8.

11. Soubrier M, Zerkak D, Gossec L, et al. Which variables best predict change in rheumatoid arthritis therapy in daily clinical practice? J Rheumatol 2006;33:1243-6.

12. Moreland LW, Weinblatt ME, Keystone EC, et al. Etanercept treatment in adults with established rheumatoid arthritis: 7 years of clinical experience. J Rheumatol 2006;33:854-61.

13. Dessein PH, Joffe BI, Singh S. Biomarkers of endothelial dysfunction, cardiovascular risk factors and atherosclerosis in rheumatoid arthritis. Arthritis Res Ther 2005; 7:R634-43.

14. Pahor A, Hojs R, Gorenjak M, et al. Accelerated atherosclerosis in pre-menopausal female patients with rheumatoid arthritis. Rheumatol Int 2006;27:119-23.

15. Georgiadis AN, Papavasiliou EC, Lourida ES, et al. Atherogenic lipid profile is a feature characteristic of patients with early rheumatoid arthritis: effect of early treatment-a prospective, controlled study. Arthritis Res Ther 2006;8:R82.

16. Wakkee M, Thio HB, Prens EP, et al. Unfavorable cardiovascular risk profiles in untreated and treated psoriasis patients. Atherosclerosis 2007;190:1-9.

17. Gelfand JM, Neimann AL, Shin DB, et al. Risk of myocardial infarction in patients with psoriasis. JAMA 2006;296:1735-41.

18. Zhang L, Astor B, Parekh R, et al. Longitudinal relationship of serum albumin and Creactive protein (CRP) with risk of cardiovascular disease (CVD) and all-cause mortality in a dialysis cohort. Circulation 2006;113:E336-7.

19. Office of National Statistics. Census 2001: National Report for England and Wales. London: The Stationery Office, 2003.

20. Currie CJ, Jones M, Goodfellow J, et al. Evaluation of survival and ischaemic and thromboembolic event rates in patients with non-valvar atrial fibrillation in the general population when treated and untreated with warfarin. Heart 2006;92:196-200.

21. Currie CJ, Morgan CL, Peters JR. Patterns and costs of hospital care for coronary heart disease related and not related to diabetes. Heart 1997:78:544-9.

22. McAlindon A, Abernethy A. Data linkage to estimate resource and service utilisation for palliative care clients. Proceedings of the symposium on health data linkage. Sydney, Australia: Australian Government Department Of Health And Ageing, 2003. http://www.publichealth.gov.au/pdf/reports_papers/symposium_procdngs_2003/ contents.pdf (accessed 16 January 2008).

23. Pearson TA, Mensah GA, Alexander WR. Markers of inflammation and cardiovascular disease: application to clinical and public health practice a statement for healthcare professionals from the Centers for Disease Control and Prevention and the American Heart Association. Circulation 2003;107:499-511. 
24. Miller M, Zhan M, Havas S. High attributable risk of elevated C-reactive protein level to conventional coronary heart disease risk factors: the Third National Health and Nutrition Examination Survey. Arch Intern Med 2005;165 2063-8.

25. Sakkinen PA, Macy EM, Callas PW, et al. Analytical and biologic variability in measures of hemostasis, fibrinolysis, and inflammation: assessment and implications for epidemiology. Am J Epidemiol 1999;149:261-7.

26. Ockene IS, Matthews CE, Rifai N, et al. Variability and classification accuracy of serial high-sensitivity $\mathrm{C}$-reactive protein measurements in healthy adults. Clin Chem 2001;47:444-50.
27. Evrin P-E, Nilsson SE, Oberg T, et al. Serum C-reactive protein in elderly men and women: association with mortality, morbidity and various biochemical values. Scand J Clin Lab Invest 2005:65:23-31.

28. Ridker PM. C-reactive protein: a simple test to help predict risk of heart attack and stroke. Circulation 2003;108:e81-5.

29. Cushman M, Arnold AM, Psaty BM, et al. C-reactive protein and the 10-year incidence of coronary heart disease in older men and women: the cardiovascula health study. Circulation 2005;112:25-31.

30. Pai JK, Pischon T, Ma J, et al. Inflammatory markers and the risk of coronary heart disease in men and women. N Engl J Med 2004;351:2599-610.

\section{Images in cardiology}

\section{Acute renal artery embolism treated with a cardiac thrombectomy catheter}

Renal artery embolism (RAE) is an uncommon but important cause of renal function loss in patients with valvular or aneurysmal cardiac disease, arrythmia or aortic atherosclerosis. Clinical diagnosis is often difficult and further invasive tests are required. Traditionally RAE has been treated with either surgical embolectomy or local thrombolysis.

A 68-year-old man with a history of previous coronary artery bypass grafting was admitted with unstable angina for cardiac catheterisation. Before the procedure he developed acute rightsided abdominal and lumbar pain associated with hypotension and oliguria. Urine analysis was positive for protein and blood. An ECG showed new atrial fibrillation. A transthoracic echocardiogram showed a left ventricular apical aneurysm with thrombus. A CT scan performed within 2 hours of pain confirmed the diagnosis of acute right renal artery occlusion (panel A). Through the right common femoral artery a 5 French Export aspiration catheter (Medtronic Vascular, Santa Rosa, CA, USA) was used to aspirate the right renal artery thrombus (panel B). There was no underlying stenotic lesion. No longterm deterioration in renal function was seen. We believe this is the first case of acute RAE treated percutaneously with a thrombus aspiration catheter.

\section{Critoph, J Radvan, D Shepherd}

chriscritoph@doctors.org.uk

Competing interests: None.

Heart 2008:94:462. doi:10.1136/hrt.2007.115618
(A) Renal angiogram showing thrombotic occlusion of the right renal artery. (B) Renal angiogram showing unobstructed right renal artery after successful percutaneous thrombus aspiration.
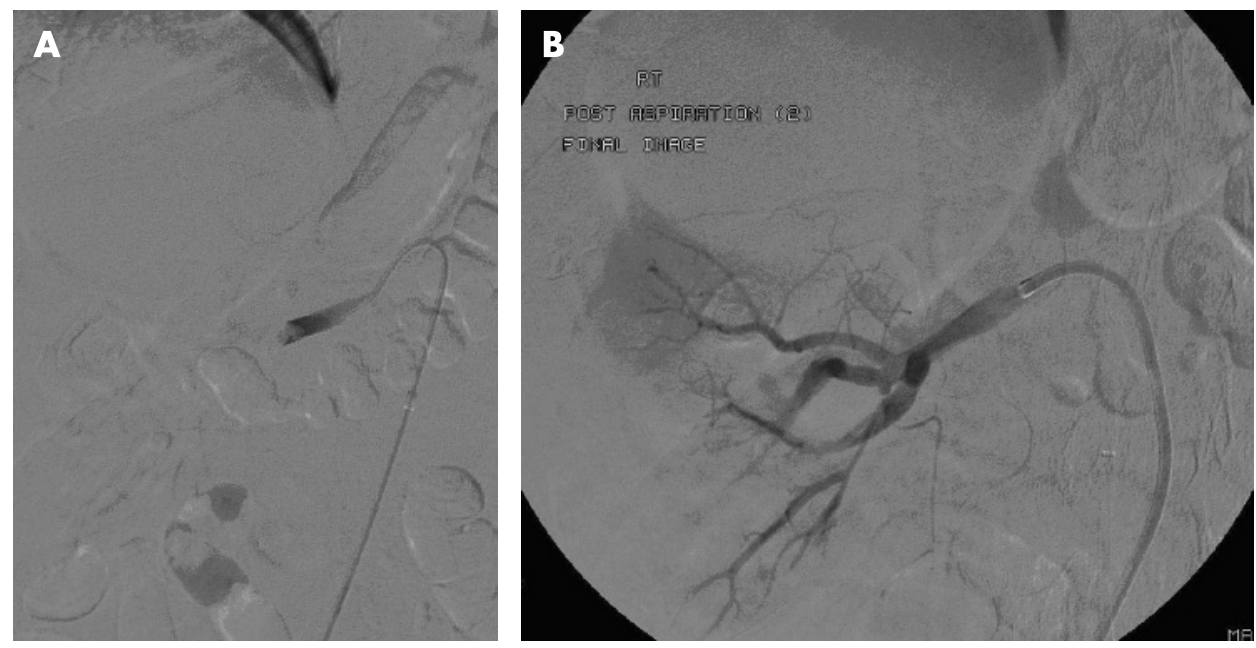